\title{
Genetic diversity analysis of yardlong bean genotypes (Vigna unguiculata subsp. sesquipedalis) based on IRAP marker
}

\author{
MUHAMMAD HABIB WIDYAWAN ${ }^{1,2, \boldsymbol{}}$, SRI WULANDARY ${ }^{1}$, TARYONO ${ }^{1,2}$ \\ ${ }^{1}$ Department of Agronomy, Faculty of Agriculture, Universitas Gadjah Mada. Jl. Flora 1, Bulaksumur, Sleman 55281, Yogyakarta, Indonesia. \\ Tel./fax.: +62-274-563062, •email: habib.widyawan@ugm.ac.id \\ ${ }^{2}$ Center of Agrotechnology Innovation, Universitas Gadjah Mada. Kalitirto, Berbah, Sleman 55573, Yogyakarta, Indonesia
}

Manuscript received: 12 November 2019. Revision accepted: 18 February 2020.

\begin{abstract}
Widyawan MH, Wulandary S, Taryono. 2020. Genetic diversity analysis of yardlong bean genotypes (Vigna unguiculata subsp. sesquipedalis) based on IRAP marker. Biodiversitas 21: 1101-1107. Inter-Retrotransposon Amplified Polymorphism (IRAP) marker is a PCR-based molecular marker that detects polymorphism between retrotransposon sites. This marker has been utilized and successfully assessed genetic diversity in many crop species. Yardlong bean (Vigna unguiculata subsp. sesquipedalis) was an important vegetable legume crop that grown mainly for its fresh pod that rich in nutritional benefits for humans, ultimately dietary fiber and protein. Trends of people awareness to nutritional content of food are increasing, therefore breeding for quality traits is important. Genetic diversity analysis is an important and elementary step in breeding programs in order to determine the breeding strategy. The aims of this research are to perform an optimization of IRAP marker and applied it for genetic diversity analysis in 16 yardlong bean genotypes. Seven primers were used as marker in a pair or single combination and resulted in 11 optimized markers that able to be used for genetic diversity analysis. Sixteen yardlong bean genotypes consisting of commercial cultivars and local genotypes from Indonesia were genotyped using eleven IRAP markers. Marker polymorphism and diversity parameters from each marker i.e. Percentage of Polymorphic Loci (PPL), Expected Heterozygosity (He), Polymorphic Information Content (PIC), Effective Multiplex Ratio (EMR), Marker Index (MI), Discriminating Power (D), and Resolving Power (RP) were calculated. Based on those values, several markers used in this study were considered as informative and efficient in terms of analyzing genetic diversity in yardlong bean. Jaccard's method was used to measure genetic similarity and it is revealed that there is high level of similarity between yardlong bean genotypes used in this study. Thus, it is implied that there is a narrow genetic diversity of yardlong bean genotypes used in this study. Cluster analysis was performed to construct dendrogram based on genetic similarity and classified 16 yardlong genotypes into 4 clusters. Interestingly, majority of the clusters formed were not able to classified genotypes based on their origin. The result of cluster analysis then confirmed by Principal Coordinate Analysis (PCoA) that able to explain $47.76 \%$ of total variation. The results of this study provide a foundation for the genetic diversity analysis based on IRAP marker and genetic improvement in yardlong bean.
\end{abstract}

Keywords: Genetic diversity, IRAP, retrotransposon, yardlong bean

\section{INTRODUCTION}

Successful crop genetic improvement by breeding program relies on the availability of broad genetic diversity. At the beginning of plant breeding program, analysis of genetic diversity among germplasms is very important. Vast amount of genetic diversity in the crop of interest enables plant breeders to select germplasms with favorable traits as the foundation for the development of new cultivars. Evaluation of genetic diversity in crop plants was conducted regularly on several basis i.e. morphological, biochemical, and molecular marker. Genetic diversity based on morphology is the simplest and the most widely used method compared to the others. However, it has several drawbacks due to its environmental effects and growth phase-dependent nature (Govindaraj et al. 2015). In several decades, molecular marker based on DNA polymorphism such as Random Amplified Polymorphic DNA (RAPD) (Williams et al. 1990), Simple Sequence Repeat (SSR), Inter Simple Sequence Repeat (ISSR), Sequence Related Amplified Polymorphism (SRAP) (Li and Quiros 2001) were gaining more attention for the analysis of genetic diversity. Mostly, it was due to their stability, abundance, and detectable modes of inheritance (Singh and Singh 2015).

Today, there are various DNA markers that available and successfully applied to assess the genetic diversity in many crop species. The analysis of genetic diversity requires molecular marker that has an ability to 'capture' the variability from polymorphism in the genome of organism. Retrotransposon is a class of Transposable Element (TE) that has an ability to move from one location to the other over the genome using a copy-and-paste mechanism (Todorovska 2007). The dispersal, abundance, and frequency of retrotransposon in plant genomes provide an excellent foundation for the development of DNA marker for genetic diversity analysis. Detection of polymorphism generated by retrotransposon insertion can be detected by Inter-Retrotransposon Amplified Polymorphism (IRAP) markers system. So far, IRAP has been applied successfully to analyze genetic diversity of various crops i.e. banana (Teo et al. 2005), rice (Branco et al. 2007), alfalfa (Mandoulakani et al. 2015), sunflower (Basirnia et 
al. 2016), cowpea (Otwe et al. 2017), and cotton (Sheidai et al. 2018).

Yardlong bean (Vigna unguiculata subsp. sesquipedalis $)(2 n=2 x=22)$, also known as asparagus bean, string bean, bodi, bora, or sitao, is known as the vegetable legume producing young immature pod for consumption. Pod produced by this plant is considered as one of the most important vegetable crops in Asia, mostly in China, East Asia, and Southeast Asia. It acts as important source of dietary fiber, proteins, amino acids, vitamins, and minerals (Kongjaimun et al. 2012). Compared to the other Chinese vegetable yardlong bean produced the highest amount of dietary fiber and protein (Wills et al. 1984). The popularity of yardlong bean is predicted to rise since people are more aware of the nutrient content of food. Thus, genetic improvement is needed to increase the quality and productivity of yardlong bean to meet the demand. To support the genetic improvement program an information about molecular marker that has an ability to assess genetic diversity of yardlong bean is needed. The aims of this study were to optimize IRAP markers and applied them for analyzing genetic diversity of 16 yardlong genotypes from germplasm collection. The ability of each marker to assess genetic diversity and overall genetic parameters were calculated and discussed. Multivariate analysis i.e. cluster analysis and Principal Coordinate Analysis (PCoA) was performed to discriminate genotypes based on their genetic similarity. The results of this study will lay a foundation of diversity analysis at molecular level for the genetic improvement of yardlong bean.

\section{MATERIALS AND METHODS}

\section{IRAP primers}

Seven IRAP primers namely NIKITA, SUKKULA, 5'LTR1, 5'LTR2, 3'LTR, LTR6149, and LTR6150 were used in this study. Those primers were used as marker in single or in paired combinations with another. List of primer names, primer sequence, length, and predicted melting temperature were listed in Table 1.

\section{Genetic materials}

Sixteen yardlong bean consists of 13 genotypes and 3 commercial cultivars were used in this study. Materials used in this study were obtained from Center of Agro Technology Innovation, Gadjah Mada University Gene Bank. Detailed information about yardlong bean used in this study was tabulated in Table 2.

\section{Genomic DNA isolation}

Genomic DNA of yardlong bean plants was isolated from fully expanded leaf taken from plants aged 21 Days After Sowing (DAS) using CTAB method (Murray and Thompson 1980) followed by RNAseA (Fermentas, Thermo Fisher Scientific) addition. Measurement of genomic DNA quality and quantity was done using agarose gel electrophoresis $(1.5 \mathrm{w} / \mathrm{v})$ and NanoDrop ${ }^{\mathrm{TM}}$ spectrophotometer. Genomic DNA then diluted into 40 $\mathrm{ng} / \mu \mathrm{L}$ concentration of working solution.

\section{PCR condition optimization}

Amplification of DNA fragment was conducted in T100 thermal cycler (BioRad). PCR profile was as follows: pre denaturation at $94{ }^{\circ} \mathrm{C}$ for 4 minutes, followed by 35 cycles of denaturation at $94{ }^{\circ} \mathrm{C}$ for 1 minute, annealing at optimum temperature for each marker for 1 minute, and elongation at $72{ }^{\circ} \mathrm{C}$ for 2 minutes, lastly, final elongation was conducted at $72{ }^{\circ} \mathrm{C}$ for 10 minutes. PCR reaction was done on $12.5 \mu \mathrm{L}$ of final volume consist of $6.25 \mu \mathrm{L}$ GoTaq ${ }^{\circledR}$ Green Master Mix (Promega), $1 \mu \mathrm{L}$ of each primer $(10 \mu \mathrm{M}), 40 \mathrm{ng}$ of DNA template, and nuclease free water up to final volume. The results of PCR were electrophoresed on $1.5 \%(\mathrm{w} / \mathrm{v})$ agarose gel.

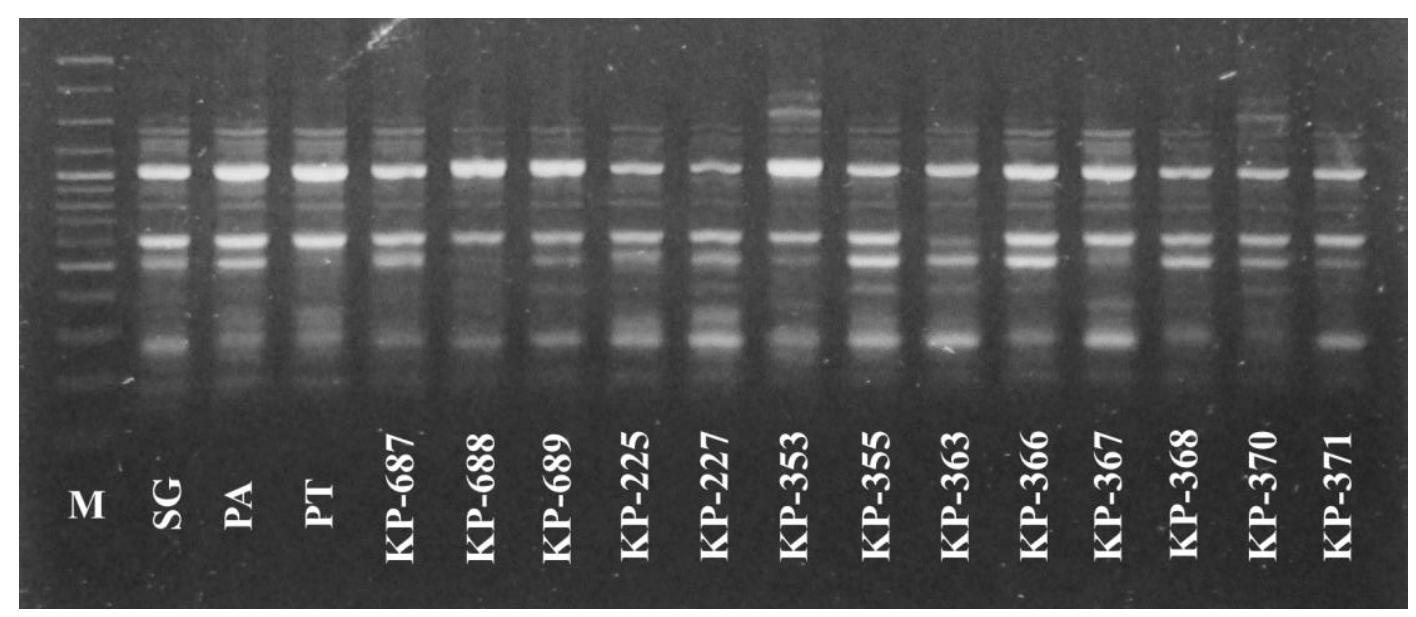

Figure 1. Result of DNA profile generation in 16 yardlong bean genotypes using Sukkula. Note: $M=100$ bp DNA ladder, letter below the DNA band in each row indicates the name of genotypes 
Table 1. Complete list of primer names, sequence, length, predicted melting temperature, and annealing temperature from primers used in this study

\begin{tabular}{clccc}
\hline Primer name & \multicolumn{1}{c}{ Primer sequence } & Length & Predicted Tm $\left({ }^{\circ} \mathbf{C}\right)$ & References \\
\hline Nikita & CGCATTTGTTCAAGCCTAAACC & 22 & 59.8 & Taheri et al. (2018) \\
Sukkula & GATAGGGTCGCATCTTGGGCGTGAC & 25 & 67.5 & Taheri et al. (2018) \\
5'LTR1 & TTGCCTCTAGGGCATATTTCCAACA & 25 & 62.7 & Taheri et al. (2018) \\
5'LTR2 & ATCATTCCCTCTAGGGCATAATTC & 24 & 58.7 & Taheri et al. (2018) \\
3'LTR & TGTTTCCCATGCGACGTTCCCCAACA & 26 & 68.8 & Taheri et al. (2018) \\
LTR6150 & CTGGTTCGGCCCATGTCTATGTATCCACACATGTA & 35 & 67.5 & Kalendar et al. (1999) \\
LTR6149 & CTCGCTCGCCCACTACTACAACCGCGTTTATT & 32 & 68.9 & Kalendar et al. (1999) \\
\hline
\end{tabular}

Note: $\mathrm{T}_{\mathrm{m}}$ of primers were predicted using IDT $^{\circledR}$ OligoAnalyzer (https://sg.idtdna.com/calc/analyzer/)

\section{Data analysis}

Products of amplification were scored based on their absence or presence of the DNA band i.e. 0 for absence and 1 for presence. To ensure the reproducibility, only clear and unambiguous DNA bands that were considered for data analysis following criteria according to Krishna et al. (2018) (DNA band data were stored in a matrix and used for the analysis of genetic diversity using iMEC (Online Marker Efficiency Calculator) (Amiryousefi et al. 2018). Total amplified loci (TAL), total polymorphic loci (TPL), percentage of polymorphic loci (PPL), expected heterozygosity $(H E)$, polymorphic information content (PIC), effective multiplex ratio (EMR), marker index (MI), discriminating power (DP), and resolving power (RP) were calculated.

Coefficient of genetic similarity between yardlong bean genotypes used in this study was estimated by Jaccard's coefficient. Clustering analysis was performed using Unweighted Paired Group Method (UPGMA) based on genetic similarity coefficients generated previously. Principal Coordinate Analysis (PCoA) was performed to confirm the result of cluster analysis. NTSYS software version 2.02 (Exeter) was used to calculate genetic similarity, cluster analysis, and Principal Coordinate Analysis.

\section{RESULTS AND DISCUSSION}

\section{Optimization of IRAP marker}

Twenty-eight IRAP markers from 7 primers were optimized using gradient PCR technique using annealing temperature ranged from $40-55{ }^{\circ} \mathrm{C}$. The best temperature i.e. resulted in clear DNA profiles with polymorphic band and clear intensity was chosen. Interestingly, temperature that results in a good DNA profile was ranged from 40.0 to 49.2 ${ }^{\circ} \mathrm{C}$, considerably deviated from the predicted annealing temperature based on calculation which ranged from 53.7-63.9 ${ }^{\circ} \mathrm{C}$. Eleven IRAP markers that able to produce DNA profiles have consisted of four markers from single primers (Nikita, Sukkula, 3'LTR, and LTR6150) and seven markers from paired primers. Markers that did not produce DNA profile at all were 5'LTR1 and 5'LTR2. On the other hand, markers that contain 5'LTR1 and 5'LTR2 primers were producing the same DNA profile as if they were used as single primers. Figure 1 showed an example of DNA amplification results from 16 genotypes using Sukkula marker.

In this present study, not all of the markers used in this study were able to generate DNA profile for analyzing genetic diversity. Kalendar and Schulman (2006) identified various problems that might lead to no amplification of the IRAP marker i.e. primer structures, competition to the target sites, and the genome composition of organism. Too small number of retrotransposon target sites or too far distance between target sites might result in no amplification. Two primers that not produce any DNA band in this study i.e. 5'LTR1 and 5'LTR2 also act in the same manner when it used on cowpea, which is closest relative of yardlong bean (Otwe et al. 2017). This finding suggests that the retrotransposon site targeted by those primers could be either present in not detectable amount or even not present in Vigna subspecies. Primer that not generated DNA band under IRAP system might could be performed better in the other retrotransposon based marker system, i.e. Retrotransposon-Microsatellite Amplified Polymorphism system (REMAP). As reported by Taheri et al. (2018) in Triticum urartu and Triticum boeoticum, primer namely LTR7286 did not produce DNA band under IRAP system. On the opposite, when the same primer combined with microsatellite primer it produced highest number of polymorphic loci.

Primers used for IRAP markers are universal since it was designed based on LTR sequences, which is highly conserved among organisms. Therefore, care should be taken for successful PCR amplification. IRAP markers are designed to amplify retrotransposon polymorphism flanked by LTR sequence. To increase the specificity of IRAP markers, the length or primers were increased up to 35 nucleotides. On the other hand, long primers will increase the annealing temperature in PCR, which in some cases can lead to failed PCR amplification. Several strategies can be applied to increase the efficiency of PCR amplification using universal primer i.e. lowering the temperature (Sergeant et al. 2012), this strategy worked successfully in our case. Based on optimization results, IRAP markers were successfully applied to analyze genetic diversity of yardlong bean in this study. 
Table 2. Details of yardlong bean accessions used in this study

\begin{tabular}{lll}
\hline ID & Name & Origin \\
\hline SG & Super Green & Commercial cultivar (Chia Tai Seeds) \\
PA & Pangeran Anvi & Commercial cultivar (Agri Makmur Pertiwi) \\
PT & Parade Tavi & Commercial cultivar (Parade Tavi (East West Seeds) \\
KP-687 & Yardlong Bean 444 & Thailand \\
KP-688 & Purple Yardlong Bean & Thailand \\
KP-689 & Golden Dragon 202 & Thailand \\
KP-225 & Sukoharjo-2 & Sukoharjo, Central Java \\
KP-227 & Sukoharjo-4 & Sukoharjo, Central Java \\
KP-353 & Purbalingga & Purbalingga, Central Java \\
KP-355 & Purbalingga-B3 & Purbalingga, Central Java \\
KP-363 & Purbalingga-D4 & Purbalingga, Central Java \\
KP-366 & Prambanan-A2 & Prambanan, Special Region of Yogyakarta \\
KP-367 & Prambanan-B & Prambanan, Special Region of Yogyakarta \\
KP-368 & Prambanan-C1 & Prambanan, Special Region of Yogyakarta \\
KP-370 & Prambanan-C3 & Prambanan, Special Region of Yogyakarta \\
KP-371 & Prambanan-4 & Prambanan, Special Region of Yogyakarta \\
\hline
\end{tabular}

\section{Marker polymorphism and diversity}

In this present study polymorphism of IRAP markers was calculated and measured (Table 3). Total 97 loci were amplified using 11 markers consisting of 4 markers from single primers and 7 markers from combination of two primers. An average of total amplified loci observed in this study was 9, moreover, Sukkula/LTR6150 marker produced the highest number of amplified loci (15) while 3'LTR marker produced the lowest number of amplified loci (2). Loci amplified by markers in this study were classified into two categories, i.e. monomorphic and polymorphic. Two single markers i.e. Nikita and 3'LTR produced monomorphic band in all loci. Percentage of polymorphic loci in this study was ranging from 0 to $66.67 \%$, with average of $45 \%$. Two markers, i.e. Nikita/Sukkula and Nikita/LTR6150 produced the highest percentage of polymorphic loci while the lowest was obtained from Nikita and 3'LTR markers.

Expected heterozygosity $(H E)$ value was calculated from 9 markers that produced polymorphic band. On average, the $H E$ value was 0.2821 , the highest value was obtained from Sukkula/LTR6150 marker (0.4592) and the lowest value was obtained from Nikita/3'LTR (0.0177). Polymorphic information content (PIC) was ranging from 0.0175 to 0.3538 , with an average of 0.2227 . Marker that produces the highest and lowest PIC was similar to those markers that produce highest and lowest $H E$. The value of average effective multiplex ratio (E) was 6.5625 , with the highest value was 10.750 (Sukkula/LTR6150) and the lowest was 2 (3'LTR). An average value of marker index (MI) obtained in this study was 0.0121 , the highest value was produced by Nikita/LTR6150 and Sukkula (0.0185) and the lowest was Nikita/3'LTR (0.0011). Discriminating power (DP) from markers used in this study was ranging from 0.0179 to 0.6118 with an average of 0.3483 . Lastly, resolving power (R) of marker was calculated. An average value of RP was 2.2386 , the lowest value was produced by Nikita/3'LTR (0.1250) and Sukkula (4.8750) produced the highest value.
The number of polymorphic loci obtained in this study was lower compared to the results reported in their closest relatives, cowpea (Otwe et al. 2017). Expected heterozygosity $(H E)$ indicates the probability of individuals that having heterozygous genotype in all loci. $H E$ also is known as genetic diversity according to $\mathrm{Nei}$ and Roychoudhury (1974), higher value indicates broader genetic diversity. The average of $H E$ value in this study was higher compared to the other study using IRAP marker from several crop species i.e. alfalfa (0.165) (Mandoulakani et al. 2015) and lower than in wheat (0.35) (Nasri et al. 2013) and sunflower (Basirnia et al. 2016). Compared to the other marker systems that have been reported on yardlong bean, IRAP provided higher value of marker informativeness, indicated by the PIC value.

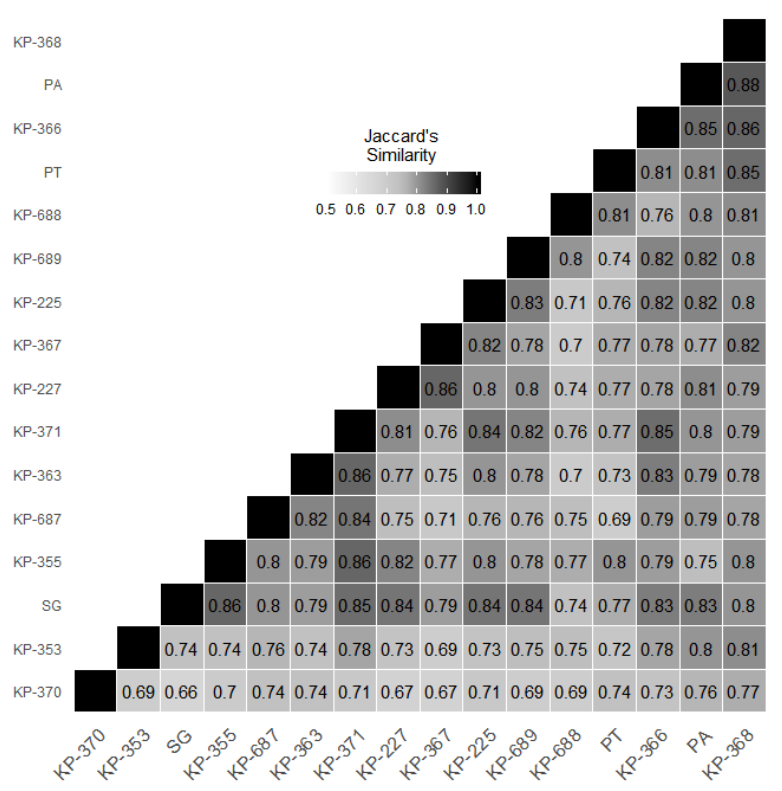

Figure 2. Visualization of Jaccard's genetic similarity coefficient among 16 yardlong bean genotypes 


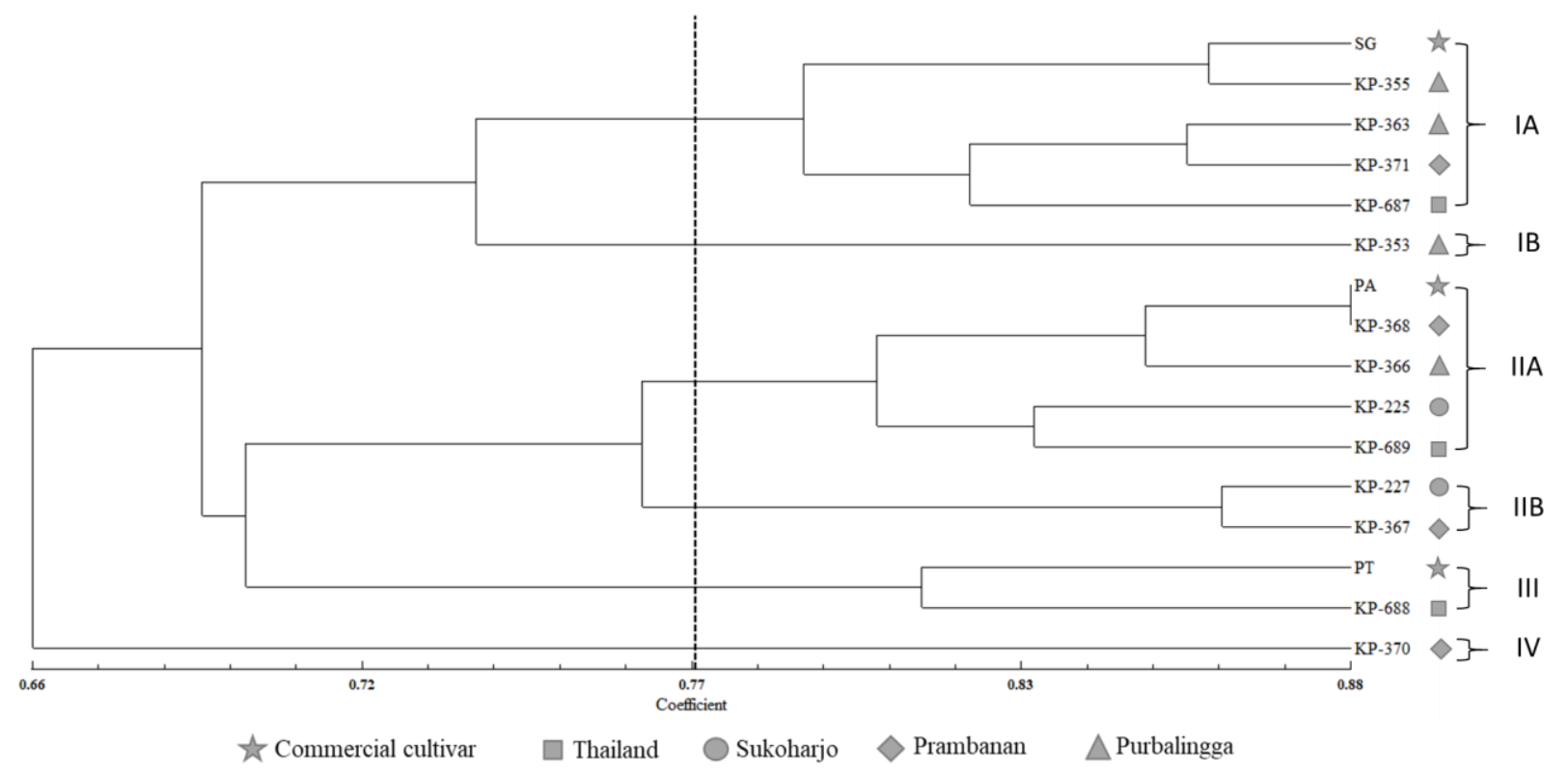

Figure 3. Dendrogram of 16 yardlong bean accessions based on IRAP marker. Note: Origin of each genotype in dendrogram was indicated by symbol on the right side of genotypes ID

The value of PIC from ISSR marker reported by Tantasawat et al. (2010) was lower than the value observed in this study. Higher PIC value indicates higher ability of molecular marker to detect allele diversity in an organism. Using the same classification according to Botstein et al. (1980), the average PIC value in this study would be classified as reasonably informative. On the other hand, genetic diversity obtained in this study was considered narrow. This might be caused by low variability that presence during the domestication yardlong bean from wild cowpea. Yardlong bean was domesticated from cowpea in Asia that domesticated from wild cowpea in Africa (Kongjaimun et al. 2012).

Associated values i.e. effective multiplex ratio, marker index, discriminating power, and resolving power were also calculated in this study (Powel et al. 1996; Nagaraju et al. 2001; Chesnokov and Artemyeva 2015). Those values were useful to assess the ability of each marker in assisting genetic diversity study. The EMR value indicates the efficiency of marker that being used, the higher EMR value marker is said to be more efficient (Chesnokov and Artemyeva 2015). It is indicated that five markers i.e. Nikita/3'LTR, Nikita/LTR6150, Sukkula, Sukkula/LTR6149, and 6150 were considered to be more efficient compared to the other markers since they possess EMR value higher than the average. The total utility of marker system was estimated using MI, the higher MI value the better the marker is and vice versa. Individuals in a population might possess different genetic profile, DP value measure the probability of two random individuals have different patterns and discriminate from one to another. Genetic diversity analysis often involves large number of germplasm, marker should be able to distinguish large number of individuals. The RP value was useful to identify marker that has an ability to analyze genetic diversity in large number of individuals. Overall, some IRAP markers in this study possess a high value of PIC, EMR, MI, DP, and RP, except markers that generated monomorphic band i.e. Nikita and 3'LTR. Thus, those IRAP markers were considered useful for analyzing genetic diversity of yardlong bean.

\section{Cluster analysis and Principal Coordinate Analysis (PCoA)}

Cluster analysis was performed to generate dendrogram based on the data of IRAP markers used in this study. Jaccard's similarity coefficient was used to establish the dendrogram. Similarity coefficient from 16 yardlong bean genotypes was ranging from $0.66-0.88$, with an average of 0.78 . Visualization of genetic similarity coefficient among yardlong bean genotypes was presented in Figure 2. The dendrogram produced was shown in Figure 3. Using the average value of similarity coefficient, a cutoff line was established. Result showed that dendrogram divided 16 yardlong bean accessions into four major clusters i.e. cluster I, II, III, and IV. The first cluster, with the largest members, consists of 6 genotypes. Moreover, it was separated into two sub-clusters i.e. IA and IB. The second cluster consists of 7 genotypes and divided into two subgroups i.e. IIA and IIB which consists of 5 and 2 genotypes, respectively. The third and fourth clusters were possessed least number of members i.e. 2 and 1 genotypes, respectively. Figure 4 showed a biplot generated by Principal Coordinate Analysis that able to explain $47.76 \%$ of total variation. The first three axes explained $21.13 \%$, $14.88 \%$, and $11.75 \%$ respectively. Two groups were established based on three-dimensional plot, the first group consists of 15 genotypes and the second group consist of only 1 genotype only, i.e. KP-370. 
Table 3. Total Amplified Loci (TAL), Total Polymorphic Loci (TPL), Percentage of Polymorphic Loci (PPL), Heterozygosity Index $(H E)$, Polymorphic Information Content (PIC), Effective Multiplex Ratio (EMR), Marker Index (MI), Discriminating Power (DP), and Resolving Power (RP) of IRAP markers used in this study

\begin{tabular}{lccccccccc}
\hline \multicolumn{1}{c}{ Marker } & TAL & TPL & PPL & HE & PIC & EMR & MI & DP & RP \\
\hline Nikita & 3 & 0 & 0.00 & 0 & 0 & 3.0000 & 0 & 0 & 0 \\
Nikita/Sukkula & 9 & 6 & 66.67 & 0.4073 & 0.3244 & 6.4375 & 0.0182 & 0.4898 & 2.3750 \\
Nikita/3'LTR & 7 & 1 & 14.29 & 0.0177 & 0.0175 & 6.9375 & 0.0011 & 0.0179 & 0.1250 \\
Nikita/LTR6150 & 15 & 10 & 66.67 & 0.4444 & 0.3457 & 10.0000 & 0.0185 & 0.5565 & 3.2500 \\
Sukkula & 14 & 9 & 64.29 & 0.4394 & 0.3428 & 9.4375 & 0.0185 & 0.5466 & 4.8750 \\
Sukkula/3'LTR & 6 & 3 & 50.00 & 0.0799 & 0.0767 & 5.7500 & 0.0048 & 0.0820 & 0.5000 \\
Sukkula/LTR6150 & 7 & 4 & 57.14 & 0.4592 & 0.3538 & 4.5000 & 0.0184 & 0.5888 & 2.7500 \\
Sukkula/LTR6149 & 14 & 7 & 50.00 & 0.3565 & 0.2930 & 10.7500 & 0.0171 & 0.4112 & 2.2500 \\
3'LTR & 2 & 0 & 0.00 & 0 & 0 & 2.0000 & 0 & 0 & 0 \\
3'LTR/LTR6150 & 6 & 3 & 50.00 & 0.4688 & 0.3589 & 3.7500 & 0.0183 & 0.6118 & 1.2500 \\
LTR6150 & 14 & 10 & 71.43 & 0.4297 & 0.3374 & 9.6250 & 0.0185 & 0.5283 & 7.2500 \\
Average & 9 & 5 & 45 & 0.2821 & 0.2227 & 6.5625 & 0.0121 & 0.3484 & 2.2386 \\
Standard deviation & 4.7078 & 3.8162 & 26.8761 & 0.2074 & 0.1601 & 3.0575 & 0.0086 & 0.2625 & 2.2772 \\
\hline
\end{tabular}

Jaccard's coefficient of similiarity reveal high similarity among yardlong bean genotypes based on IRAP marker genotyping. This finding confirmed the low number of polymorphic loci and genetic diversity value obtained in this study. Thus, it suggest that those yardlong bean genotypes might share similar morphological features although further study is needed. Dendrogram generated using IRAP marker classified 16 yardlong bean genotypes into four clusters. Three-yardlong bean cultivars i.e. SG, PA, and PT were used as check in this study based on their good agronomical traits and popularity in Indonesia and belong to different clusters i.e. IA, IIA, and III respectively. There are genotypes located close to each check in, i.e. SG and KP-355, PA and KP-386, and PT with KP-688, indicated the similar genetic features that they shared. Clearly, they possess similar genetic similarities and might share similar phenotypic features, which is favorable for breeding programs. Three-dimensional plot generated by PCoA confirmed the result of cluster analysis (Figure 4). Moreover, it is revealed that yardlong bean germplasm used in this study were classified into two groups. The first group with the largest number of members possess genotypes from cluster I, II, and III and the second group only consist of one genotype i.e. KP-370 that classified in the cluster IV.

Interestingly, only two genotypes from the same origin belong to the same cluster i.e. KP-355 and KP-363 from Purbalingga in the first cluster. However, they were placed on different sub-cluster. These findings indicate that they might be derived from same parental in the past and altered through selection. IRAP marker was reported to be less powerful in terms of distinguishing genotypes based on their origin. Taheri et al. (2018) reported that IRAP marker was not able to separate Triticum uratu and T. boeoticum genotypes properly based on their geographical distribution. Retrotransposon activities in plant are believed to be triggered by stress-induced by environmental conditions (Lanciano et al. 2017, Nie et al. 2019). In general, yardlong bean germplasm used in this study was originated from Southeast Asia i.e. Thailand and mostly Indonesia. Those countries are classified as tropical countries and possess similar environmental properties. Thus, similar pattern of insertional-mutational activity of retrotransposon might have occurred.

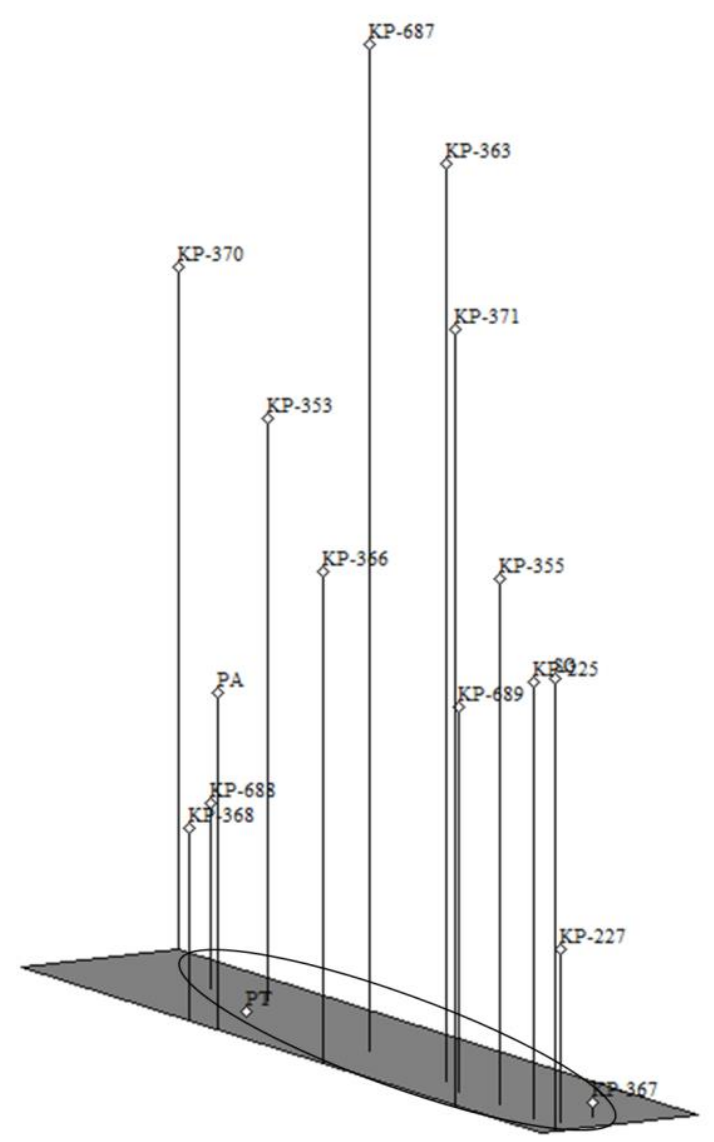

Figure 4. Three-dimensional plot from Principle Coordinate Analysis (PCoA) of 16 yardlong bean genotypes. Note: The circle on three-dimensional plot indicates the first group. 
Overall, IRAP markers that useful for genetic diversity analysis of yardlong bean were identified. Those markers will be useful for further diversity analysis at molecular level in yardlong bean. Their capability to analyze genetic diversity also comparable to the other marker system available. Narrow genetic diversity of yardlong bean genotypes were revealed in this study, compared to the other species. Thus, it will limits breeders to select genotypes that suitable for breeding programs. Effort to increase the genetic diversity of yardlong bean should be performed. There are various options available to increase variability in plant breeding programs, from the conventional to the cutting edge methods involving molecular techniques. Narrow genetic diversity of yardlong bean is supposed to be caused by its domestication. Therefore, a technique that able to induce novel phenotypes that not present previously in yardlong bean is preferable. Mutation technique using chemical or physical agents could suit that objective. New traits that not available in nature could emerge as the implication of random mutational activity. Thus, it will increase the genetic diversity of yardlong bean and support the genetic improvement program.

\section{ACKNOWLEDGEMENTS}

Gadjah Mada University Young Scientist Capacity Building Grant 2019 sponsored this study. Authors would like to say thanks to Dr. Agus Budi Setiawan (Gadjah Mada University) for the fruitful discussion and Rr. Sri Rakhmi Sayekti, M.Sc. (Center of Agro Innovation Gadjah Mada University) for her technical assistance during the preparation of the genetic materials.

\section{REFERENCES}

Amiryousefi A, Hyvonen J, Poczai P. 2018. iMEC: online marker efficiency calculator. App Plant Sci 6: 1-4.

Basirnia A, Darvishzadeh R, Mandoulkani BA. 2016. Retrotransposon insertional polymorphism in sunflower (Helianthus annuus L.) lines revealed by IRAP and REMAP markers. Plant Biosyst 150: 641-651.

Botstein D, White RL, Skolnick M, Davis RW. 1980. Construction of a genetic linkage map in man using restriction fragment length polymorphism. Am J Hum Genet 32: 314-331.

Branco CJS, Vieira EAm Malone G, Kopp MM, Malone E, Bernarndes A, Mistura CC, Carvalho FIF, Oliveira CA. 2007. IRAP and REMAP assessments of genetic similarity in rice. J Appl Genet 48: 107-113.

Chesnokov YV, Artemyva AM. 2015. Evaluation of the measure of polymorphism information on genetic diversity. Agr Biol 50: 571578.

Govindaraj M, Vetriventhan M, Srinivasan M. 2015. Importance of genetic diversity assessment in crop plants and its recent advances: an overview of its analytical perspectives. Genet Res Int 2015: 1-14.

Kalendar R, Grob T, Regina M, Suoniemi A, Schulman A. 1999. IRAP and REMAP: two new retrotransposon-based DNA fingerprinting techniques. Theor Appl Genet 98: 704-711.

Kalendar R, Schulman AH. 2006. IRAP and REMAP for retrotransposonbased genotyping and fingerprinting. Nat Prot 5: 2478-2484.

Kongjaimun A, Kaga A, Tomooka N, Somta P, Vaughan DA, Srinives P 2012. The genetics of domestication of yardlong bean, Vigna unguiculata (L.) Walp. ssp. unguiculata cv.-gr. Sesquipedalis. Ann Bot 109: 1185-1200.

Krishna TPA, Maharajan T, David RHA, Ramakrishnan M, Ceasar SA, Duraipandiyan V, Roch GV, Ignacimuthu S. 2018. Microsatellite markers of finger millet (Eleusine coracana (L.) Gaertn) and foxtail millet (Setaria italica (L.) Beauv) provides resources for crossgenome transferability and genetic diversity analysis in other millets. Biocatal Agric Biotechnol 16: 493-501.

Lanciano S, Carpentier M, Llauro C, Jobet E, Robakowska-Hyzorek D, Lassere E, Ghesquiere A, Panaud O, Mirouzae M. 2017. Sequencing the extrachromosomal circular mobilome reveals retrotransposon activity in plants. PLoS Genet 13(2): e1006630. DOI: 10.1371/journal.pgen.1006630.

Li G, Quiros CF. 2001. Sequence-related amplified polymorphism (SRAP), a new marker system based on a simple PCR reaction: its application to mapping and gene tagging in Brassica. Theor Appl Genet 103: 455-461.

Mandoulakani BA, Sadigh P, Azizi H, Piri Y, Nasri S, Arzahang S. 2015. Comparative assessment of IRAP, REMAP, ISSR, and SSR markers for evaluation of genetic diversity of alfalfa (Medicago sativa $\mathrm{L}$.). J Agr Sci Tech 17: 999-1010.

Murray MG, Thompson WF. 1980. Rapid isolation of high molecular weight DNA. Nuc Acid Res 8: 4321-4325.

Nagaraju J, Damodar Reddy K, Nagaraja GM, Sethuraman BN. 2001. Comparison of multilocus RFLP and PCR-based marker systems for genetic analysis of silkworm, Bombyx mori. Heredity 86: 588-597.

Nasri S, Mandoulakani BA, Darvishzadeh R, Bernousi L. 2013. Retrotransposon insertional polymorphism in Iranian bread wheat cultivars and breeding lines revealed by IRAP and REMAP markers. Biochem Genet 51: 927-943.

Nei M, Roychoudhury AK. 1974. Sampling variances of heterozygosity of genetic distance. Genetics 76: 379-390.

Nie Q, Qiao G, Peng L, Wen X. 2019. Transcriptional activation of long terminal repeats retrotransposon sequences in the genome of pitaya under abiotic stress. Plant Phys Biochem 135: 460-468.

Otwe EP, Agyirifo DS, Galyuon IK, Heslop-Harrison JS. 2017. Molecular diversity in some Ghanaian cowpea [Vigna unguiculata L. (Walp)] accessions. Trop Plant Biol 10: 57-67.

Powell W, Morgante M, Andre C, Hanafey M, Vogel J, Tingey S, Rafalski A. 1996. The comparison of RFLP, RAPD, AFLP, and SSR (microsatellite) markers for germplasm analysis. Mol Breed 2: 225230.

Sergeant MJ, Constantinidou C, Cogan T, Penn CW, Pallen MJ. 2012. Hight-throughput sequencing of $16 \mathrm{~s}$ rRNA gene amplicons: effects of extraction procedure, primer length, and annealing temperature. PLoS One 7: e38094. DOI: 10.1371/journal.pone.0038094.

Sheidai M, Riazifar M, Hoordadian A, Alishah O. 2018. Genetic fingerprinting of salt and drought-tolerant cotton cultivars (Gossypium hirsutum) by IRAP-REMAP and SRAP molecular marker. Plant Gene 14: 12-19.

Singh BD, Singh AK. 2015. Marker-assisted plant breeding: principles and practices. Springer, New Delhi.

Tantasawat P, Trongchuen J, Prajongjai T, Seehalak W, Jittayasothorn Y. 2010. Variety identification and comparative analysis of genetic diversity in yardlong bean (Vigna unguiculata spp. sesquipedalis) using morphological characters, SSR, and ISSR analysis. Sci Hort 125: 204-216.

Taheri MT, Alavi-Kia SS, Mohammadi SA, Vahed MM. 2018. Assessment of genetic diversity and relationships among Triticum urartu and Triticum boeoticum populations from Iran using IRAP and REMAP markers. Genet Resour Crop Evol 65:1867-1878.

Teo CH, Tan SH, Ho CL, Faridah QZ, Othman YR, Heslop-Harrison JS, Kalendar R, Schulman AH. 2005. Genome constitution and classification using retrotransposon-based marker in the orphan crop banana. J Plant Biol 48: 96-105.

Todorovska E. 2007. Retrotransposons and their role in plant-genome evolution. Biotechnol Biotechnol Eq 21: 294-305.

Williams JG, Kubelik AR, Livak KJ, Rafalski JA, Tingey SV. 1990. DNA polymorphism amplified by arbitrary primers are useful as genetic marker. Nuc Acids Res 25: 6531-6535.

Wills RBH, Wong AWK, Scriven FM, Greenfield H. 1984. Nutrient composition of Chinese vegetables. J Agric Food Chem 32: 414-416. 